\title{
Optimal Sobolev and Hardy-Rellich constants under Navier boundary conditions
}

\author{
Filippo Gazzola, Hans-Christoph Grunau†, Guido Sweers ${ }^{\ddagger}$
}

\begin{abstract}
We prove that the best constant for the critical embedding of higher order Sobolev spaces does not depend on all the traces. The proof uses a comparison principle due to Talenti [19] and an extension argument which enables us to extend radial functions from the ball to the whole space with no increase of the Dirichlet norm. Similar arguments may also be used to prove the very same result for Hardy-Rellich inequalities.
\end{abstract}

AMS Classification: primary 46E35, secondary 26D10, $35 \mathrm{J55}$

Keywords: optimal constant, Sobolev embedding, Hardy-Rellich inequality

\section{Introduction and results}

Let $n \in \mathbb{N}, p \in(1, \infty)$ and $m \in \mathbb{N}$ with $m<n / p$. Then, it is well-known [1] that the Sobolev space $W^{m, p}\left(\mathbb{R}^{n}\right)$ embeds continuously into $L^{n p /(n-m p)}\left(\mathbb{R}^{n}\right)$. Since $p^{*}:=n p /(n-m p)$ is the largest exponent for which embeddings into $L^{q}\left(\mathbb{R}^{n}\right)$ spaces hold, it is called the critical Sobolev exponent. In fact, more refined embeddings hold true. On the space $C_{c}^{\infty}\left(\mathbb{R}^{n}\right)$ of smooth compactly supported functions, define the norm

$$
\|u\|_{m, p, \mathbb{R}^{n}}:= \begin{cases}\left\|\Delta^{k} u\right\|_{L^{p}\left(\mathbb{R}^{n}\right)} & \text { if } m=2 k, \\ \left\|\nabla\left(\Delta^{k} u\right)\right\|_{L^{p}\left(\mathbb{R}^{n}\right)}:=\left\|\left|\nabla\left(\Delta^{k} u\right)\right|\right\|_{L^{p}\left(\mathbb{R}^{n}\right)} & \text { if } m=2 k+1,\end{cases}
$$

and denote by $\mathcal{D}^{m, p}\left(\mathbb{R}^{n}\right)$ the closure of $C_{c}^{\infty}\left(\mathbb{R}^{n}\right)$ with respect to the norm (1). Then, also the larger space $\mathcal{D}^{m, p}\left(\mathbb{R}^{n}\right)$ embeds continuously into $L^{p^{*}}\left(\mathbb{R}^{n}\right)$ and a best Sobolev constant for the corresponding embedding is defined, see $[12,18]$ and also previous results in [20]. More precisely, let

$$
S_{m, p}:=\inf _{\mathcal{D}^{m, p}\left(\mathbb{R}^{n}\right) \backslash\{0\}} \frac{\|u\|_{m, p, \mathbb{R}^{n}}^{p}}{\|u\|_{L^{p^{*}}\left(\mathbb{R}^{n}\right)}^{p}},
$$

then $S_{m, p}>0$ and

$$
S_{m, p}\|u\|_{L^{p^{*}\left(\mathbb{R}^{n}\right)}}^{p} \leq\|u\|_{m, p, \mathbb{R}^{n}}^{p} \quad \text { for all } u \in \mathcal{D}^{m, p}\left(\mathbb{R}^{n}\right) .
$$

It can be shown that the infimum in (2) is achieved and, when $m=1$ or $p=2$, the constant $S_{m, p}$ can be explicitly computed, see $[12,17,18,20]$.

Similar results are also available in bounded domains. Let $\Omega \subset \mathbb{R}^{n}$ be a bounded domain and on the space $C_{c}^{\infty}(\Omega)$ of smooth compactly supported functions, define the norm

$$
\|u\|_{m, p, \Omega}:= \begin{cases}\left\|\Delta^{k} u\right\|_{L^{p}(\Omega)} & \text { if } m=2 k, \\ \left\|\nabla\left(\Delta^{k} u\right)\right\|_{L^{p}(\Omega)}:=\left\|\left|\nabla\left(\Delta^{k} u\right)\right|\right\|_{L^{p}(\Omega)} & \text { if } m=2 k+1,\end{cases}
$$

\footnotetext{
*Dipartimento di Matematica, Politecnico di Milano, Italy

${ }^{\dagger}$ Institut für Analysis und Numerik, Otto-von-Guericke-Universität Magdeburg, Germany

${ }^{\ddagger}$ Mathematisches Institut, Universität zu Köln, Germany
} 
and denote by $W_{0}^{m, p}(\Omega)$ the closure of $C_{c}^{\infty}(\Omega)$ with respect to the norm $\|\cdot\|_{m, p, \Omega}$ from (3). Again, one is interested in the optimal constant for the embedding inequality. By taking advantage of scaling arguments and the concentration-compactness principle, it is shown in [12] that

$$
\inf _{W_{0}^{m, p}(\Omega) \backslash\{0\}} \frac{\|u\|_{m, p, \Omega}^{p}}{\|u\|_{L^{p^{*}}(\Omega)}^{p}}=S_{m, p},
$$

where $S_{m, p}$ is the same constant as in (2). In other words, the best Sobolev constant for the critical embedding is independent of the domain. However, contrary to the case addressed in (2), the infimum in (4) is not achieved.

The space $W_{0}^{m, p}(\Omega)$ may also be seen as the closed subspace of $W^{m, p}(\Omega)$ of functions with vanishing traces up to order $m-1$. A natural question which arises is to find out whether the best embedding constant (4) depends on all the traces. The space of particular interest that appears, is

$$
W_{\vartheta}^{m, p}(\Omega):=\left\{v \in W^{m, p}(\Omega) ; \Delta^{j} v_{\mid \partial \Omega}=0 \text { in the sense of traces for } 0 \leq j<\frac{m}{2}\right\} .
$$

Indeed, when $p=2$, the space $W_{\vartheta}^{m, 2}(\Omega)$ is the space where variational solutions to polyharmonic elliptic pde's are sought when complemented with the so-called homogenous Navier boundary conditions on $\partial \Omega$. With these boundary conditions, the polyharmonic equation may be rewritten as a second order system. Note that $W_{\vartheta}^{m, p}(\Omega)$ strictly contains $W_{0}^{m, p}(\Omega)$ so that the just mentioned question is of interest. The first step to answer this question is to define a suitable norm and, in this case, some smoothness of the boundary is required. For simplicity, we take $\partial \Omega \in C^{m}$ although much less regularity is needed, see [2]. In such case, $\|\cdot\|_{m, p, \Omega}$ from (3) is a complete norm also on $W_{\vartheta}^{m, p}(\Omega)$. Then, the best embedding constant is defined by

$$
S_{m, p, \vartheta}(\Omega):=\inf _{W_{\vartheta}^{m, p}(\Omega) \backslash\{0\}} \frac{\|u\|_{m, p, \Omega}^{p}}{\|u\|_{L^{p^{*}}(\Omega)}^{p}} .
$$

In view of the just mentioned inclusion $W_{0}^{m, p}(\Omega) \subset W_{\vartheta}^{m, p}(\Omega)$, it is clear that $S_{m, p, \vartheta}(\Omega) \leq S_{m, p}$ for any bounded smooth domain $\Omega$.

One then wonders whether this inequality is even an equality and, subsequently, whether $S_{m, p, \vartheta}(\Omega)$ is independent of the domain $\Omega$. When $m=p=2$, this question was answered positively in a paper by van der Vorst [21]. Subsequently, Ge [9] gave a positive answer when $p=2$ for any $m \in \mathbb{N}$. As far as we are aware, the general case $p>1$ has not been previously considered. Both papers $[9,21]$ are based on the concentration-compactness principle by Lions [12]. Van der Vorst [21, p.259] claims that "the concentration-compactness lemma is a virtual transcription of the principle due to Lions" although a crucial part of the proof is not carried out in full detail. However, it is not clear to us how the result in [21, Lemma A1] can be proved with an extension argument as in [12] since a) the space $C_{c}^{\infty}(\Omega)$ is not dense in $W_{\vartheta}^{m, p}(\Omega)$ and b) functions in $W_{\vartheta}^{m, p}(\Omega)$ cannot be trivially extended to $\mathbb{R}^{n}$.

The first purpose of the present paper is to provide a complete proof to the following statement:

Theorem 1. Let $n \in \mathbb{N}, p \in(1, \infty)$ and $m \in \mathbb{N}$ with $m<n / p$. Let $\Omega \subset \mathbb{R}^{n}$ be a bounded domain with $\partial \Omega \in C^{m}$. Then,

$$
S_{m, p, \vartheta}(\Omega)=S_{m, p} .
$$

This result is in striking contrast with subcritical embeddings for which the best embedding constant does depend on the traces, see [7]. Moreover, according to Talenti [20], "if $p=1$ the Sobolev inequality behaves in a slightly different manner" and Cassani-Ruf-Tarsi [5] show that Theorem 1 becomes false for $p=1$. 
We prove Theorem 1 with the aid of two basic tools. First, we use a comparison principle due to Talenti [19] to reduce it to the case where $\Omega$ is a ball and the infimum in (6) is taken among positive radially symmetric functions, see Section 2. Second, we show how such functions may be extended to $\mathbb{R}^{n}$ with no increase of the $W_{\vartheta}^{m, p}$-norm and with increase of the $L^{p^{*}}$-norm, see Section 3.

In fact, these tools may be used to prove similar results also for the Hardy-Rellich inequalities which can be considered as classical Sobolev-type embedding inequalities. The first version of these inequalities appears in $[10,11]$ whereas a higher order generalization has been proved by Rellich [16]. More recently, further extensions have appeared in $[6,14]$, see also [8] for inequalities with remainder terms. Although it is not explicitly assumed that $0 \in \Omega$, here we have this particularly interesting case in mind. Assume again that $p>1$ and $m<n / p$. Then, there exists a constant $H_{m, p}>0$, independent of $\Omega$, such that

$$
\inf _{W_{0}^{m, p}(\Omega) \backslash\{0\}} \frac{\|u\|_{m, p, \Omega}^{p}}{\int_{\Omega} \frac{|u(x)|^{p}}{|x|^{m p}} d x}=H_{m, p} .
$$

For this result and the exact value of $H_{m, p}$ we refer to [6, Corollary 14]. Then, by arguing as in the proof of Theorem 1 one obtains

Theorem 2. Let $n \in \mathbb{N}, p \in(1, \infty)$ and $m \in \mathbb{N}$ with $m<n / p$. Let $\Omega \subset \mathbb{R}^{n}$ be a bounded domain with $\partial \Omega \in C^{m}$. Then,

$$
\inf _{W_{\vartheta}^{m, p}(\Omega) \backslash\{0\}} \frac{\|u\|_{m, p, \Omega}^{p}}{\int_{\Omega} \frac{\mid u\left(\left.x\right|^{p}\right.}{|x|^{m p}} d x}=H_{m, p} .
$$

There are only two small differences between proving Theorems 1 and 2. First, contrary to (2), the best Hardy-Rellich constant $H_{m, p}$ is not attained on $\mathbb{R}^{n}$. However, this gives no further complication in the proof. Second, in order to reduce to the radial situation, beside Talenti's principle one should also recall that symmetrization increases $L^{p}$-norms with the singular weight $|x|^{-m p}$, see [3, Theorem 2.2].

This paper is organized as follows. In Section 2 we recall a comparison principle due to Talenti [19] and we explain how it may be used in the proof of Theorem 1. In Section 3 we explain and comment the main ideas of the proof of Theorem 1; to this end, we give a simple proof in the case $m=2$. In Section 4 we give the complete proof of Theorem 1 for any $m \geq 2$.

\section{The iterated Talenti principle}

Here and in the sequel, we denote by $B$ the unit ball, by $e_{n}=|B|$ its measure and by $f^{*} \in L^{p}(B)$ the spherical rearrangement of $f \in L^{p}(\Omega)$ according to [19, p. 701] when $|\Omega|=|B|$ and $p>1$. We remark that in particular $f^{*}=|f|^{*}$. A crucial tool for the proof of Theorem 1 is the following comparison principle due to Talenti [19, Theorem 1].

Proposition 3. Let $\Omega \subset \mathbb{R}^{n}$ ( $n \geq 2$ ) be a $C^{m}$-smooth bounded domain such that $|\Omega|=|B|=e_{n}$. Let $q \geq 2 n /(n+2)$ and let $m=2 k$ be an even number. Let $f \in L^{q}(\Omega)$ and let $u \in W_{\vartheta}^{m, q}(\Omega)$ be the unique strong solution to

$$
\begin{cases}(-\Delta)^{k} u=f & \text { in } \Omega, \\ \Delta^{j} u=0 & \text { on } \partial \Omega, \quad j=0, \ldots, k-1 .\end{cases}
$$


Let $f^{*} \in L^{q}(B)$ and $u^{*} \in W_{0}^{1, q}(B)$ denote respectively the spherical rearrangements of $f$ and $u$, and let $v \in W_{\vartheta}^{m, q}(B)$ be the unique strong solution to

$$
\begin{cases}(-\Delta)^{k} v=f^{*} & \text { in } B, \\ \Delta^{j} v=0 & \text { on } \partial B, \quad j=0, \ldots, k-1 .\end{cases}
$$

Then, $v \geq u^{*}$ a.e. in $B$.

Remark 4. In this proposition we assumed that $\partial \Omega \in C^{m}$. In order to use the result of Talenti it is sufficient that $\partial \Omega \in C^{1,1}$. Indeed, this condition guarantees that the solution of the Dirichlet Laplacian with right hand side in $L^{p}(\Omega)$ exists uniquely in $W_{\vartheta}^{2, p}(\Omega)$. For such a boundary however, the solution of $(7)$ in general does not lie in $W_{\vartheta}^{m, p}(\Omega)$ but in another space, which can be defined for even $m$ as

$$
\widetilde{W}_{\vartheta}^{m, p}(\Omega):=\left\{v \in W_{\vartheta}^{2, p}(\Omega) ; \Delta^{j} v \in W_{\vartheta}^{2, p}(\Omega) \text { for } 0 \leq j<\frac{m}{2}\right\} .
$$

If one drops the smoothness assumption even further, the result does not hold true any longer with the same generality. For $q=m=2$ there is still a unique weak solution of $\Delta^{2} u=f$ in the sense of a weak 'system' solution, namely $u, u_{1} \in W_{0}^{1,2}(\Omega)$ satisfying weakly $-\Delta u_{1}=f$ and $-\Delta u=u_{1}$ but in general $u \notin W^{2,2}(\Omega)$. It shows that on nonsmooth domains $\Omega$ the map $u \mapsto\|u\|_{2,2, \Omega}$ is not a norm in $W_{\vartheta}^{2,2}(\Omega)$. Moreover, there might be a weak 'equation' solution $\tilde{u} \neq u$ with $\tilde{u} \in W_{\vartheta}^{2,2}(\Omega)$ and nevertheless $\Delta \tilde{u}=0$ pointwise almost everywhere on $\partial \Omega$, see [15].

Proof. When $k=1$ Proposition 3 is precisely [19, Theorem 1]. For $k \geq 2$ we proceed by finite induction. We may rewrite (7) and (8) as the following systems:

$$
\begin{array}{ll}
\left\{\begin{array}{lll}
-\Delta u_{1}=f & \text { in } \Omega, \\
u_{1}=0 & \text { on } \partial \Omega,
\end{array}\right. & \begin{cases}-\Delta u_{i}=u_{i-1} & \text { in } \Omega, \\
u_{i}=0 & \text { on } \partial \Omega,\end{cases} \\
\begin{cases}-\Delta v_{1}=f^{*} & \text { in } B, \\
v_{1}=0 & \text { on } \partial B,\end{cases} & \begin{cases}-\Delta v_{i}=v_{i-1} & \text { in } B, \\
v_{i}=0 & \text { on } \partial B,\end{cases}
\end{array}
$$

Note that $u_{k}=u$ and $v_{k}=v$. By Talenti's principle [19, Theorem 1] applied for $i=1$, we know that $v_{1} \geq u_{1}^{*}$ a.e. in $B$. Assume that the inequality $v_{i} \geq u_{i}^{*}$ a.e. in $B$ has been proved for some $i=1, \ldots, k-1$. Then, by (10) and (11) we infer

$$
\left\{\begin{array} { l l } 
{ - \Delta u _ { i + 1 } = u _ { i } } & { \text { in } \Omega } \\
{ u _ { i + 1 } = 0 } & { \text { on } \partial \Omega , }
\end{array} \quad \left\{\begin{array}{ll}
-\Delta v_{i+1}=v_{i} \geq u_{i}^{*} & \text { in } B \\
v_{i+1}=0 & \text { on } \partial B .
\end{array}\right.\right.
$$

By combining the maximum principle for $-\Delta$ in $B$ with a further application of Talenti's principle, we obtain $v_{i+1} \geq u_{i+1}^{*}$ a.e. in $B$. This finite induction shows that $v_{k} \geq u_{k}^{*}$ and proves the statement.

Let us now explain how we intend to apply Proposition 3. Assume that $m$ is even, $m=2 k$ for some $k \in \mathbb{N}$, and note that $S_{m, p, \vartheta}(\Omega)$ is invariant under scaling so that we may assume that $\Omega$ has the same measure as the unit ball, $|\Omega|=|B|=e_{n}$. In order to apply the iterated Talenti principle we further fix

$$
\bar{q}:=\frac{2 n}{n+2} .
$$

If $p \geq \bar{q}$ we know that $W_{\vartheta}^{m, p}(\Omega) \subset W_{\vartheta}^{m, \bar{q}}(\Omega)$ but if $p<\bar{q}$ this inclusion fails. For any $u \in$ $W_{\vartheta}^{m, p}(\Omega) \cap W_{\vartheta}^{m, \bar{q}}(\Omega)$, consider $v \in W_{\vartheta}^{m, p}(B) \cap W_{\vartheta}^{m, \bar{q}}(B)$ given by

$$
\begin{cases}(-\Delta)^{k} v=\left((-\Delta)^{k} u\right)^{*} & \text { in } B \\ \Delta^{j} v=0 & \text { on } \partial B, \quad j=0, \ldots, k-1 .\end{cases}
$$


Then, $(-\Delta)^{j} v$ is positive, radially symmetric and radially decreasing for all $j=0, \ldots, k$. Moreover, Proposition 3 yields $v \geq u^{*}$ so that

$$
\|v\|_{L^{p^{*}(B)}} \geq\left\|u^{*}\right\|_{L^{p^{*}(B)}}=\|u\|_{L^{p^{*}}(\Omega)},
$$

where the last equality follows from standard properties of symmetric rearrangements, see e.g. [3]. For the same reason, we also have

$$
\left\|\Delta^{k} v\right\|_{L^{p}(B)}=\left\|\left(\Delta^{k} u\right)^{*}\right\|_{L^{p}(B)}=\left\|\Delta^{k} u\right\|_{L^{p}(\Omega)}
$$

and we obtain the following statement:

Lemma 5. Let $p>1$ and let $m=2 k$ be even. Then, for any $u \in W_{\vartheta}^{m, p}(\Omega) \cap W_{\vartheta}^{m, \bar{q}}(\Omega)$ there exists a positive radial function $v \in W_{\vartheta}^{m, p}(B) \cap W_{\vartheta}^{m, \bar{q}}(B)$ (i.e. $v=v(r), r=|x|$ ) such that

$$
r \mapsto(-\Delta)^{j} v(r) \quad \text { is positive and radially decreasing for all } 0 \leq j \leq k
$$

and

$$
\frac{\|u\|_{m, p, \Omega}^{p}}{\|u\|_{L^{p^{*}(\Omega)}}^{p}} \geq \frac{\|v\|_{m, p, B}^{p}}{\|v\|_{L^{p^{*}(B)}}^{p}} .
$$

When $m$ is odd, $m=2 k+1$ for some $k \geq 1$, the same result may be obtained with slightly more work. For any $u \in W_{\vartheta}^{m, p}(\Omega)$ consider again $v \in W_{\vartheta}^{m, p}(B)$ defined by (12). Then, $(-\Delta)^{j} v$ is positive, radially symmetric and radially decreasing for all $j=0, \ldots, k$. Moreover, we obtain again (13). Finally, by standard properties of symmetric rearrangements [3], we infer

$$
\left\|\nabla\left(\Delta^{k} v\right)\right\|_{L^{p}(B)}=\left\|\nabla\left(\Delta^{k} u\right)^{*}\right\|_{L^{p}(B)} \leq\left\|\nabla\left(\Delta^{k} u\right)\right\|_{L^{p}(\Omega)}
$$

and we obtain:

Lemma 6. Let $p>1$ and let $m=2 k+1$ be odd. Then, for any $u \in W_{\vartheta}^{m, p}(\Omega) \cap W_{\vartheta}^{m, \bar{q}}(\Omega)$ there exists a positive radial function $v \in W_{\vartheta}^{m, p}(B) \cap W_{\vartheta}^{m, \bar{q}}(B)$ (i.e. $v=v(r), r=|x|$ ) such that (14) and (15) hold.

Let $p>1, m$ be any integer and consider minimizing sequences for $S_{m, p, \vartheta}(\Omega)$ in $W_{\vartheta}^{m, p}(\Omega)$. Since $\Omega$ is a bounded smooth domain, smooth functions can be shown to be dense in $W_{\vartheta}^{m, p}(\Omega)$ by referring to the existence theory for strong solutions of Navier boundary value problems. This means that we may restrict ourselves to minimizing sequences for $S_{m, p, \vartheta}(\Omega)$ in $W_{\vartheta}^{m, p}(\Omega) \cap$ $W_{\vartheta}^{m, \bar{q}}(\Omega)$. Lemmas 5 and 6 now show that

$$
\inf _{R_{\vartheta}^{m, p}(B) \backslash\{0\}} \frac{\|u\|_{m, p, B}^{p}}{\|u\|_{L^{p^{*}(B)}}^{p}} \leq S_{m, p, \vartheta}(B) \leq S_{m, p, \vartheta}(\Omega),
$$

where $R_{\vartheta}^{m, p}(B)$ denotes the positive convex cone of $W_{\vartheta}^{m, p}(B)$ containing radially symmetric functions $v$ such that (14) holds. Moreover, as already mentioned in the previous section, we have $S_{m, p, \vartheta}(\Omega) \leq S_{m, p}$ for any domain $\Omega$. Hence, we have

$$
\inf _{R_{\vartheta}^{m, p}(B) \backslash\{0\}} \frac{\|u\|_{m, p, B}^{p}}{\|u\|_{L^{p^{*}(B)}}^{p}} \leq S_{m, p, \vartheta}(B) \leq S_{m, p, \vartheta}(\Omega) \leq S_{m, p}
$$

for all $\Omega$ and the proof of Theorem 1 is complete (for any $m$ ) if we show that

$$
\inf _{R_{\vartheta}^{m, p}(B) \backslash\{0\}} \frac{\|u\|_{m, p, B}^{p}}{\|u\|_{L^{p^{*}(B)}}^{p}} \geq S_{m, p} .
$$




\section{The extension argument and comments}

In this section we will give a proof of Theorem 1 for the simplest case.

Proof for $m=2$. As already mentioned, Theorem 1 follows if we prove (16). We assume that $m=2$ and, for contradiction, also that there exists $u \in R_{\vartheta}^{2, p}(B) \backslash\{0\}$ such that

$$
\frac{\|u\|_{m, p, B}^{p}}{\|u\|_{L^{p^{*}(B)}}^{p}}<S_{2, p}
$$

Note that necessarily $u^{\prime}(1)<0$ since $u^{\prime}(1)=0$ would imply $u \in W_{0}^{2,2}(B)$ contradicting (4). Consider the radial entire function defined by

$$
w(r):= \begin{cases}u(r)+\frac{1}{n-2}\left|u^{\prime}(1)\right| & \text { for } r \in(0,1], \\ \frac{r^{2-n}}{n-2}\left|u^{\prime}(1)\right| & \text { for } r \in[1, \infty),\end{cases}
$$

and note that $w \in C^{1,1}(0, \infty)$ and $w \in \mathcal{D}^{2, p}\left(\mathbb{R}^{n}\right)$. Moreover, $\|w\|_{L^{p^{*}}\left(\mathbb{R}^{n}\right)}>\|u\|_{L^{p^{*}}(B)}$ whereas $\|\Delta w\|_{L^{p}\left(\mathbb{R}^{n}\right)}=\|\Delta u\|_{L^{p}(B)}$. Hence, by (17),

$$
\frac{\|w\|_{m, p, \mathbb{R}^{n}}^{p}}{\|w\|_{L^{p^{*}\left(\mathbb{R}^{n}\right)}}^{p}}<\frac{\|u\|_{m, p, B}^{p}}{\|u\|_{L^{p^{*}(B)}}^{p}}<S_{2, p}
$$

which contradicts (2).

In the remaining of this section we comment the above proof in the simplest Hilbertian case $p=2$. It consists of three steps. First, Talenti's comparison principle enables us to restrict our attention to the case where $\Omega=B$ and $u$ is radially symmetric. Then, by contradiction, if (17) holds we may build an entire function by increasing the $L^{2^{*}}$-norm and maintaining the $L^{2}$-norm of its Laplacian, since the modification is performed by adding a constant in $B$ and a multiple of the fundamental solution outside $B$. This extension is possible for any $u \in R_{\vartheta}^{2,2}(B) \backslash\{0\}$ and the increase of its $L^{2^{*}}$-norm may be estimated as follows:

$$
\begin{aligned}
& \|w\|_{L^{2^{*}\left(\mathbb{R}^{n}\right)}}^{2^{*}}=\left\|u+C_{n}\left|u^{\prime}(1)\right|\right\|_{L^{2^{*}(B)}}^{2^{*}}+\|w\|_{L^{2^{*}}\left(\mathbb{R}^{n} \backslash B\right)}^{2^{*}} \cdots \\
& \ldots\left\{\begin{array}{l}
<\left(\|u\|_{L^{2^{*}(B)}}+C_{n}\left|u^{\prime}(1)\right|\right)^{2^{*}}+C_{n}\left|u^{\prime}(1)\right|^{2^{*}}, \\
>\|u\|_{L^{2^{*}(B)}}^{2^{*}}+C_{n}\left|u^{\prime}(1)\right|^{2^{*}},
\end{array}\right.
\end{aligned}
$$

where $C_{n}$ are positive constants which may differ also within the same line. The two above inequalities show that $\left|u^{\prime}(1)\right|$ measures "the increase of the $L^{2^{*}}$-norm" and large values of $\left|u^{\prime}(1)\right|$ correspond to large increases of the norm.

For any $\varepsilon>0$ consider the entire functions

$$
u_{\varepsilon}(x):=\frac{\varepsilon^{n-4}}{\left(\varepsilon^{2}+|x|^{2}\right)^{\frac{n-4}{2}}}
$$

which all satisfy $u_{\varepsilon}(0)=1$. It is known (see e.g. [12]) that they achieve the best constant in (2), that is,

$$
S_{2,2}=\frac{\left\|u_{\varepsilon}\right\|_{2,2, \mathbb{R}^{n}}^{2}}{\left\|u_{\varepsilon}\right\|_{L^{2^{*}}\left(\mathbb{R}^{n}\right)}^{2}} \quad \text { for all } \varepsilon>0 .
$$

We "lower" $u_{\varepsilon}$ by setting

$$
U_{\varepsilon}(x):=u_{\varepsilon}(x)-\frac{\varepsilon^{n-4}}{\left(\varepsilon^{2}+1\right)^{\frac{n-4}{2}}}=\frac{\varepsilon^{n-4}}{\left(\varepsilon^{2}+|x|^{2}\right)^{\frac{n-4}{2}}}-\frac{\varepsilon^{n-4}}{\left(\varepsilon^{2}+1\right)^{\frac{n-4}{2}}}
$$


so that $U_{\varepsilon} \in W_{\vartheta}^{2,2}(B)$ and one can show that (see [4])

$$
\lim _{\varepsilon \rightarrow 0} \frac{\left\|\Delta U_{\varepsilon}\right\|_{L^{2}(B)}^{2}}{\left\|U_{\varepsilon}\right\|_{L^{2^{*}(B)}}^{2}}=S_{2,2} .
$$

In this respect, $\left|U_{\varepsilon}^{\prime}(1)\right|=\frac{(n-4) \varepsilon^{n-4}}{\left(1+\varepsilon^{2}\right)^{(n-2) / 2}}$ is a measure of concentration. If it is small then $\varepsilon$ is small, which corresponds to high concentration (Sobolev ratio close to $S_{2,2}$ ). In this case, we have seen

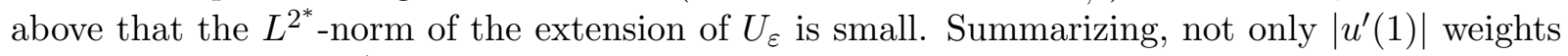
the increase of the $L^{2^{*}}$-norm of the extension of $u$ but also its degree of concentration.

A similar but slightly more delicate interpretation can be given for all $m$ and $p$. As we shall see in next section, in this case there are more extension parameters. Since the norm of the extension is related to the degree of concentration, it seems that all these parameters could possibly reduce to only one or at least just a few. This gives rise to a further question. Does the optimal Sobolev constant depend only on the first trace(s)? In other words, is $S_{m, p}$ also the best constant for the embedding

$$
W_{\mathcal{B}}^{m, p}(\Omega)=\left\{W^{m, p}(\Omega) ; \mathcal{B}(u)=0\right\} \subset L^{p^{*}}(\Omega) ?
$$

Here $\mathcal{B}(u)$ is a set of at least $\left[\frac{m+1}{2}\right]$ boundary conditions which guarantee that $\|\cdot\|_{m, p, \Omega}$ is a norm on $W_{\mathcal{B}}^{m, p}(\Omega)$. Maz'ya [13, Section 1.6.6] proves that for $W^{m, p}(\Omega) \cap W_{0}^{\ell, p}(\Omega)$ with $2 \ell \geq m$ embeddings only need the boundedness of $\Omega$. If $W_{\mathcal{B}}^{m, p}(\Omega) \not \subset W^{m, p}(\Omega) \cap W_{0}^{\ell, p}(\Omega)$ for some $\ell \geq \frac{1}{2} m$, that is, if not all first $\left[\frac{m+1}{2}\right]$-traces are contained in $\mathcal{B}$, then the regularity of the domain $\Omega$ plays a role.

\section{Proof of Theorem 1}

The full proof of Theorem 1 needs to distinguish between even and odd $m$.

Even $m, m=2 k$ for some $k \geq 1$.

For $g:[0,1] \rightarrow \mathbb{R}$ let us define

$$
\left(\mathcal{G}_{0} g\right)(r):=\int_{r}^{1} \int_{0}^{\rho}\left(\frac{s}{\rho}\right)^{n-1} g(s) d s d \rho
$$

Hence, $\mathcal{G}_{0}$ is the solution operator for the radially symmetric Poisson problem in the unit ball of $\mathbb{R}^{n}$, that is, it satisfies

$$
\left\{\begin{array}{cc}
-\Delta\left(\mathcal{G}_{0} g\right)(|x|)=g(|x|) & \text { for }|x|<1 \\
\left(\mathcal{G}_{0} g\right)(|x|)=0 & \text { for }|x|=1
\end{array}\right.
$$

Let us also define for $g:[0, \infty) \rightarrow \mathbb{R}$ with appropriate integrability conditions

$$
(\mathcal{G g})(r):=\int_{r}^{\infty} \int_{0}^{\rho}\left(\frac{s}{\rho}\right)^{n-1} g(s) d s d \rho
$$

If $g$ also goes to 0 fast enough for $r \rightarrow \infty$ (e.g. like $r^{-\gamma}$ with $\gamma>2$ ), then an integration by parts gives

$$
(\mathcal{G} g)(r)=\frac{1}{n-2} r^{2-n} \int_{0}^{r} s^{n-1} g(s) d s+\frac{1}{n-2} \int_{r}^{\infty} s g(s) d s,
$$

and

$$
-\Delta(\mathcal{G g})(|x|)=g(|x|) \text { for } x \in \mathbb{R}^{n}
$$

Note that

$$
g \geq 0 \Longrightarrow \mathcal{G} g \geq \mathcal{G}_{0} g \text { in } B
$$

We now describe the inductive procedure which we will use in order to suitably extend functions in $R_{\vartheta}^{m, p}(B)$. 
Lemma 7. Let $n, \gamma>2, \gamma \neq n$ and let $\ell \geq 0$. If $f \in W_{l o c}^{\ell, p}\left(\mathbb{R}^{n}\right)$ is radially symmetric, positive and such that

$$
f(x) \leq c_{f}|x|^{-\gamma} \text { for }|x|>1,
$$

then there is a unique radially symmetric solution $u \in W_{l o c}^{\ell+2, p}\left(\mathbb{R}^{n}\right)$ of

$$
\left\{\begin{array}{c}
-\Delta u=f \text { in } \mathbb{R}^{n} \\
\lim _{|x| \rightarrow \infty} u(x)=0
\end{array}\right.
$$

Moreover, $u=\mathcal{G} f$ implies that $u$ is positive and

$$
u(x) \leq c_{2}|x|^{2-n}+\frac{c_{f}}{(\gamma-2)(n-\gamma)}|x|^{2-\gamma} \text { for }|x|>1 .
$$

Equality holds if $f(x)=c_{f}|x|^{-\gamma}$ for $|x|>1$.

Proof. In view of the "boundary condition" at infinity, uniqueness follows from Weyl's lemma and Liouville's theorem.

Suppose first that $f$ is continuous. We have

$$
-r^{1-n} \partial_{r}\left(r^{n-1} \partial_{r} u(r)\right)=f(r) .
$$

Since $\partial_{r} u$ is bounded in 0 we find

$$
r^{n-1} \partial_{r} u(r)=-\int_{0}^{r} s^{n-1} f(s) d s
$$

and since $u$ goes to 0 at $\infty$ it follows that

$$
\begin{aligned}
u(r) & =\int_{r}^{\infty} \rho^{1-n} \int_{0}^{\rho} s^{n-1} f(s) d s d \rho \\
& =\frac{-1}{n-2}\left[\rho^{2-n} \int_{0}^{\rho} s^{n-1} f(s) d s\right]_{r}^{\infty}+\frac{1}{n-2} \int_{r}^{\infty} s f(s) d s \\
& =\frac{1}{n-2} r^{2-n} \int_{0}^{r} s^{n-1} f(s) d s+\frac{1}{n-2} \int_{r}^{\infty} s f(s) d s .
\end{aligned}
$$

If $f \geq 0$ is not identically 0 , then $u>0$. For $r>1$ it follows from (20) that

$$
\begin{aligned}
u(r) & \leq \frac{1}{n-2} r^{2-n}\left(\int_{0}^{1} s^{n-1} f(s) d s+c_{f} \int_{1}^{r} s^{n-1-\gamma} d s\right)+\frac{c_{f}}{(n-2)(\gamma-2)} r^{2-\gamma} \\
& =\frac{1}{n-2}\left(\int_{0}^{1} s^{n-1} f(s) d s-\frac{c_{f}}{n-\gamma}\right) r^{2-n}+\frac{c_{f}}{(\gamma-2)(n-\gamma)} r^{2-\gamma} .
\end{aligned}
$$

Equality holds if $f(x)=c_{f}|x|^{-\gamma}$ for $|x|>1$. The formula in (20) also holds for $f \in W_{l o c}^{\ell, p}\left(\mathbb{R}^{n}\right)$. The claim that $u \in W_{l o c}^{\ell+2, p}\left(\mathbb{R}^{n}\right)$ is direct.

The second tool is a variation of an extension result which enables us to modify functions in $R_{\vartheta}^{m, p}(B)$ to functions on the whole space with no increase of the Dirichlet norm.

Lemma 8. Let $m=2 k$ and let $u \in R_{\vartheta}^{m, p}(B) \backslash\{0\}$. Let $w(r)=\left(\mathcal{G}^{k} f\right)(r)$ for

$$
f(r)=\left\{\begin{array}{cl}
(-\Delta)^{k} u(r) & \text { for } r \leq 1 \\
0 & \text { for } r>1
\end{array}\right.
$$

then $w \in \mathcal{D}^{m, p}\left(\mathbb{R}^{n}\right)$ and 
1. $\|w\|_{m, p, \mathbb{R}^{n}}=\|u\|_{m, p, B}$

2. $\|w\|_{L^{p^{*}}\left(\mathbb{R}^{n}\right)}>\|u\|_{L^{p^{*}(B)}}$.

Proof. From Lemma 7 we find that

$$
w(r)=c_{1} r^{2-n}+c_{2} r^{4-n}+\cdots+c_{m} r^{m-n} \text { for } r>1
$$

which implies with $w \in W_{l o c}^{m, p}\left(\mathbb{R}^{n}\right)$ that $w \in \mathcal{D}^{m, p}\left(\mathbb{R}^{n}\right)$. Here, it is crucial that $p>1$ is assumed. Since

$$
f(r)=(-\Delta)^{k}\left(\mathcal{G}^{k} f\right)(r)=(-\Delta)^{k} w(r)
$$

it even follows that

$$
\|w\|_{m, p, \mathbb{R}^{n}}=\left\|\Delta^{k} w\right\|_{L^{p}\left(\mathbb{R}^{n}\right)}=\|f\|_{L^{p}\left(\mathbb{R}^{n}\right)}=\|f\|_{L^{p}(B)}=\left\|\Delta^{k} u\right\|_{L^{p}(\Omega)}=\|u\|_{m, p, B} .
$$

Moreover, by (18) it follows that $\mathcal{G} f(1)>0=\left((-\Delta)^{k-1} u\right)(1)$ and hence by the maximum principle and by (19)

$$
\mathcal{G} f \geq \mathcal{G}_{0} f=(-\Delta)^{k-1} u \text { in } B .
$$

Since $\mathcal{G}^{2} f(1)>0=\left((-\Delta)^{k-2} u\right)(1)$ and since $(21)$ holds, a further iteration of the maximum principle and (19) implies

$$
\mathcal{G}^{2} f \geq \mathcal{G}_{0}^{2} f=(-\Delta)^{k-2} u \text { in } B .
$$

Repeating this argument we find

$$
w=\mathcal{G}^{k} f \geq \mathcal{G}_{0}^{k} f=u \text { in } B .
$$

Hence $\|w\|_{L^{p^{*}}\left(\mathbb{R}^{n}\right)}>\|w\|_{L^{p^{*}(B)}} \geq\|u\|_{L^{p^{*}(B)}}$.

As already mentioned, the proof of Theorem 1 follows if we show that (16) holds. By contradiction, assume that there exists $u \in R_{\vartheta}^{m, p}(B) \backslash\{0\}$ such that

$$
\frac{\|u\|_{m, p, B}^{p}}{\|u\|_{L^{p^{*}(B)}}^{p}}<S_{m, p}
$$

Let $w \in \mathcal{D}^{m, p}\left(\mathbb{R}^{n}\right) \backslash\{0\}$ be the function constructed in Lemma 8 . Then, Lemma 8 shows that

$$
\frac{\|w\|_{m, p, \mathbb{R}^{n}}^{p}}{\|w\|_{L^{p^{*}}\left(\mathbb{R}^{n}\right)}^{p}}<\frac{\|u\|_{m, p, B}^{p}}{\|u\|_{L^{p^{*}(B)}}^{p}}<S_{m, p}
$$

which contradicts (2). This contradiction completes the proof of Theorem 1 for even $m=2 k$.

Odd $m, m=2 k+1$ for some $k \geq 1$. In this case, we take advantage of what has just been proved for the even exponent $2 k$. Since $W_{\vartheta}^{2 k+1, p}(B) \subset W_{\vartheta}^{2 k, p}(B)$, by Lemma 8 we know that any $u \in R_{\vartheta}^{2 k+1, p}(B) \backslash\{0\}$ allows to define an entire function $w$ such that

$$
w>u \text { in } B, \quad \Delta^{k}(w-u)=0 \text { in } B, \quad \Delta^{k} w=0 \text { in } \mathbb{R}^{n} \backslash B .
$$

In particular, this implies that also

$$
\nabla\left(\Delta^{k}(w-u)\right)=0 \text { in } B, \quad \nabla\left(\Delta^{k} w\right)=0 \text { in } \mathbb{R}^{n} \backslash B .
$$

The construction for the $2 k$-case also enables us to conclude that $w \in C^{2 k-1}\left(\mathbb{R}^{n}\right)$, a regularity which is not enough to obtain $w \in \mathcal{D}^{2 k+1, p}\left(\mathbb{R}^{n}\right)$, here we need one more degree of regularity. This is obtained by recalling the extra boundary condition that appears by going from $W_{\vartheta}^{2 k, p}(B)$ to $W_{\vartheta}^{2 k+1, p}(B)$, namely $\Delta^{k} u=0$ on $\partial B$, and that $\Delta^{k} w=0$ in $\mathbb{R}^{n} \backslash B$. 
Remark 9. The inductive procedure described in Lemmas 7 and 8 can be resolved even explicitly. For the comparison function $w$ introduced above one finds after some tedious calculations that

$$
w(r)= \begin{cases}u(r)+\sum_{\ell=0}^{k-1} a_{\ell}^{(k)} r^{2 \ell} & \text { for } r \leq 1, \\ \sum_{\ell=0}^{k-1} b_{\ell}^{(k)} r^{2 \ell+2-n} & \text { for } r>1,\end{cases}
$$

where

$$
a_{\ell}^{(k)}=\frac{(-1)^{\ell+1}}{2^{\ell} \ell ! \prod_{s=1}^{\ell}(n-2+2 s)} \cdot\left(\sum_{j=0}^{k-1-\ell} \frac{1}{j ! 2^{j} \prod_{s=0}^{j}(n-2-2 s)}\left((-\Delta)^{j+\ell} u\right)^{\prime}(1)\right)
$$

and

$$
b_{\ell}^{(k)}=\frac{1}{2^{\ell} \ell ! \prod_{s=1}^{\ell}(n-2-2 s)} \cdot\left(\sum_{j=0}^{k-1-\ell} \frac{(-1)^{j+1}}{j ! 2^{j} \prod_{s=0}^{j}(n-2+2 s)}\left((-\Delta)^{j+\ell} u\right)^{\prime}(1)\right) .
$$

Acknowledgement. We thank Daniele Cassani for raising the problem and for stimulating discussions. We also thank Andrea Cianchi for an inspiring discussion on results of Maz'ya [13] which led to the comments at the end of Section 3.

\section{References}

[1] R.A. Adams, Sobolev Spaces, Pure and Applied Mathematics 65, Academic Press, New York-London, 1975

[2] V. Adolfsson, $L^{2}$-integrability of second-order derivatives for Poisson's equation in nonsmooth domains, Math. Scand. 70, 1992, 146-160

[3] F.J. Almgren, E.H. Lieb, Symmetric decreasing rearrangement is sometimes continuous, J. Amer. Math. Soc. 2, 1989, 683-773

[4] E. Berchio, F. Gazzola, T. Weth, Critical growth biharmonic elliptic problems under Steklov-type boundary conditions, Adv. Diff. Eq. 12, 2007, 381-406

[5] D. Cassani, B. Ruf, C. Tarsi, Best constants in a borderline case of second order Moser type inequalities, to appear in Ann. Inst. H. Poincaré

[6] E.B. Davies, A.M. Hinz, Explicit constants for Rellich inequalities in $L_{p}(\Omega)$, Math. Z. 227, 1998, $511-523$

[7] A. Ferrero, F. Gazzola, T. Weth, Positivity, symmetry and uniqueness for minimizers of second-order Sobolev inequalities, Ann. Mat. Pura Appl. (4) 186, 2007, 565-578

[8] F. Gazzola, H.-Ch. Grunau, E. Mitidieri, Hardy inequalities with optimal constants and remainder terms, Trans. Amer. Math. Soc. 356, 2004, 2149-2168

[9] Y. Ge, Sharp Sobolev inequalities in critical dimensions, Michigan Math. J. 51, 2003, 27-45

[10] G.H. Hardy, Notes on some points in the integral calculus, Messenger Math. 48, 1919, 107-112

[11] G.H. Hardy, J.E. Littlewood, G. Pólya, Inequalities, Cambridge University Press, 1934 
[12] P.L. Lions, The concentration-compactness principle in the calculus of variations. The limit case. I, Rev. Mat. Iberoamericana 1, 1985, 145-201

[13] V.G. Maz'ya, Sobolev Spaces, Springer Series in Soviet Mathematics, Translated from the Russian by T.O. Shaposhnikova, Springer-Verlag, Berlin, 1985

[14] E. Mitidieri, A simple approach to Hardy's inequalities, Math. Notes 67, 2000, 479-486. Russian original: Mat. Zametki 67, 2000, 563-572

[15] S.A. Nazarov, G. Sweers, A hinged plate equation and iterated Dirichlet Laplace operator on domains with concave corners, J. Differential Equations 233, 2007, 151-180

[16] F. Rellich, Halbbeschränkte Differentialoperatoren höherer Ordnung, Proceedings of the International Congress of Mathematicians, Amsterdam 1954, vol. III, Erven P. Noordhoff N.V., Groningen, 1956, 243-250

[17] E. Rodemich, The Sobolev inequalities with best possible constants, in: Analysis Seminar at California Institute of Technology, 1966

[18] Ch.A. Swanson, The best Sobolev constant, Appl. Anal. 47, 1992, 227-239

[19] G. Talenti, Elliptic equations and rearrangements, Ann. Scuola Norm. Sup. Pisa Cl. Sci. (4) 3, 1976, 697-718

[20] G. Talenti, Best constant in Sobolev inequality, Ann. Mat. Pura Appl. (4) 110, 1976, 353-372

[21] R.C.A.M. van der Vorst, Best constant for the embedding of the space $H^{2} \cap H_{0}^{1}(\Omega)$ into $L^{2 N /(N-4)}(\Omega)$, Diff. Int. Eq. 6, 1993, 259-276 